Research Article

\title{
Fractional-Order Sliding Mode Synchronization for Fractional-Order Chaotic Systems
}

\author{
Chenhui Wang (10 \\ College of Applied Mathematics, Xiamen University of Technology, Xiamen 361024, China \\ Correspondence should be addressed to Chenhui Wang; chwang@xmut.edu.cn
}

Received 5 October 2017; Revised 19 December 2017; Accepted 20 December 2017; Published 17 January 2018

Academic Editor: Christos Volos

Copyright (C) 2018 Chenhui Wang. This is an open access article distributed under the Creative Commons Attribution License, which permits unrestricted use, distribution, and reproduction in any medium, provided the original work is properly cited.

\begin{abstract}
Some sufficient conditions, which are valid for stability check of fractional-order nonlinear systems, are given in this paper. Based on these results, the synchronization of two fractional-order chaotic systems is investigated. A novel fractional-order sliding surface, which is composed of a synchronization error and its fractional-order integral, is introduced. The asymptotical stability of the synchronization error dynamical system can be guaranteed by the proposed fractional-order sliding mode controller. Finally, two numerical examples are given to show the feasibility of the proposed methods.
\end{abstract}

\section{Introduction}

In the past two decades, synchronization of chaotic systems (CSs) has received more and more attention, and a lot of interesting works have been done, which have potential application values in secret communications, signal processing, and complex systems [1-9]. Recently, control and synchronization of fractional-order chaotic systems (FOCSs), which can be seen as a generalization of the integer-order CSs, have been studied extensively. A lot of controllers have been implemented such as active control [10], feedback control [11], sliding mode control $[12,13]$, adaptive control, $[14,15]$, and adaptive fuzzy control $[8,9,16]$.

It is well known that sliding mode control (SMC) is a very effective control method to cope with system uncertainties and external disturbances [17-27]. Consequently, it has been used to synchronize FOCSs. For example, a novel FOCS and its SMC have been studied in [28]; SMC of a 3D FOCS using a fractional-order switching type controller is investigated in [29]. Using a hierarchical fuzzy neural network, [30] proposed a new adaptive SMC method for the synchronization of uncertain FOCSs. On the other hand, it is well known that, in stability analysis of nonlinear systems, quadratic Lyapunov functions are most commonly used. However, [31,32] show that it is not realistic to use quadratic
Lyapunov functions in the stability analysis of fractionalorder nonlinear systems due to the complicated infinite series produced by differentiating the squared Lyapunov function with fractional order. It should be mentioned that, in most aforementioned works, the stability analysis is given based on fractional Lyapunov methods. How to establish some stability analysis methods according to the model of FOCSs is a meaningful work.

In control theory, stability analysis is an essential aspect. With respect to fractional-order linear systems, the stability condition was firstly investigated in [33]. Then, using LMI, some sufficient conditions are given in [34]. The related results on the stability analysis of fractional-order nonlinear systems can be seen in [35-41] and the references therein. It should be pointed out that the stability criterion for fractional-order nonlinear systems requires further study. Thus, proposing some new stability criterion for FOCSs is necessary. In this paper, we will give two sufficient conditions for the stability of a class of FOCSs. Based on these theorems, a fractional-order SMC will be given. The contributions of this paper are concluded as follows: (1) two sufficient conditions are proposed to check the stability of the fractionalorder nonlinear system and (2) a novel fractional-order SMC is given, and the stability of the closed-loop system is proven rigorously. 


\section{Preliminaries}

In this section, we will give some properties of fractional calculus. The $q$ th fractional-order integral is expressed as [42]

$$
\mathbb{D}^{q} f(t)=\frac{1}{\Gamma(q)} \int_{0}^{t}(t-\tau)^{q-1} f(\tau) d \tau
$$

The Caputo fractional derivative is given by

$$
\mathbb{D}^{q} f(t)=\frac{1}{\Gamma(n-q)} \int_{0}^{t}(t-\tau)^{n-q-1} f^{(n)}(\tau) d \tau,
$$

where $q$ is the fractional order satisfying $n-1 \leq q<n$.

The Laplace transform of Caputo fractional derivative is given as [42]

$$
\int_{0}^{\infty} e^{-s t} \mathbb{D}^{q} x(t) d t=s^{q} F(s)-\sum_{k=0}^{n-1} s^{q-k-1} x^{(k)}(0),
$$

where $F(s)=\mathscr{L}\{f(t)\}$. In the next section, we will use the following results.

The Mittag-Leffler function is given by

$$
E_{\beta_{1}, \beta_{2}}(z)=\sum_{k=0}^{\infty} \frac{z^{k}}{\Gamma\left(\beta_{1} k+\beta_{2}\right)},
$$

where $\beta_{1}, \beta_{2}>0$ and $z \in C$. The Laplace transform of (4) is

$$
\mathscr{L}\left\{t^{\beta_{2}-1} E_{\beta_{1}, \beta_{2}}\left(-a t^{\beta_{1}}\right)\right\}=\frac{s^{\beta_{1}-\beta_{2}}}{s^{\beta_{1}}+a} .
$$

Lemma 1 (see [42]). Let $A \in R^{n \times n}, 0<\alpha \leq 1, \beta$ be an arbitrary real number, and $b>0$ be a real constant; then,

$$
E_{\alpha, \beta}(A) \leq \frac{b}{1+\|A\|},
$$

where $\mu \leq|\arg (\operatorname{eig}(A))| \leq \pi$ with $\mu \in R$ satisfying $\pi \alpha / 2<\mu<$ $\min \{\pi, \pi \alpha\}$.

Lemma 2 (see $[43])$. Let $t \in[0, T]$ and

$$
x(t) \leq h(t)+\int_{0}^{t} k(\tau) x(\tau) d \tau,
$$

where $k(t) \geq 0$. Then, one has

$$
x(t) \leq h(t)+\int_{0}^{t} k(\tau) h(\tau) \exp \left[\int_{\tau}^{t} k(u) d u\right] d \tau .
$$

Lemma 3 (see $[42,44]$ ). Let $0<\alpha<2$. $\beta$ is a complex number, and $\mu$ is a real number. If

$$
\frac{\pi \alpha}{2}<\mu<\min \{\pi, \pi \alpha\}
$$

then, for an arbitrary integer $n \geq 1$, the following expansion holds:

$$
E_{\alpha, \beta}(z)=-\sum_{j=1}^{n} \frac{1}{\Gamma(\beta-\alpha j) z^{j}}+\circ\left(\frac{1}{|z|^{n+1}}\right) .
$$

\section{Main Results}

3.1. Some Sufficient Conditions for the Stability Analysis of Fractional-Order Systems. Consider a class of fractionalorder systems described by

$$
\mathbb{D}^{q} x_{j}(t)=c_{j} x_{j}(t)+\sum_{i=1}^{n} a_{i j} f_{i}\left(x_{i}(t)\right),
$$

or equivalently

$$
\mathbb{D}^{q} x(t)=C x(t)+A f(x(t)),
$$

where $j=1,2, \ldots, n, 0<q<1$, and $x(t)=$ $\left[x_{1}(t), x_{2}(t), \ldots, x_{n}(t)\right]^{T} \in \mathbb{R}^{n}$ is the state vector; $f(x(t))=$ $\left[f_{1}\left(x_{1}(t)\right), f_{2}\left(x_{2}(t)\right), \ldots, f_{n}\left(x_{n}(t)\right)\right]^{T} \in \mathbb{R}^{n}$ represents a smooth nonlinear function, $A=\left\{a_{i j}\right\}, i=1,2, \ldots, n, j=$ $1,2, \ldots, n$, and $C=\operatorname{diag}\left(c_{i}\right)$ are two matrices. Then, we have the following results.

Theorem 4. If $c_{i}<0$ and the nonlinear function is bounded, that is, there exists a constant $m_{i}>0$ such that

$$
\left|f_{i}\left(x_{i}(t)\right)\right| \leq m_{i}
$$

then there exist two positive constants $t_{0}$ and $M$ such that

$$
\left|x_{i}(t)\right| \leq M
$$

for all $t>t_{0}$

Proof. It follows from (11) that

$$
X_{i}(s)=\frac{s^{q-1}}{s^{q}-c_{i}} x_{i}(0)+\frac{1}{s^{q}-c_{i}} \sum_{j=1}^{n} a_{i j} \mathscr{L}\left(f_{j}\left(x_{j}(t)\right)\right) .
$$

Using (5), one solves (15) as

$$
\begin{aligned}
& x_{i}(t)=x_{i}(0) E_{q, 1}\left(c_{i} t^{q}\right)+\sum_{j=1}^{n} a_{i j} \\
& \cdot \int_{0}^{t}(t-\tau)^{q-1} E_{q, q}\left(c_{i}(t-\tau)^{q}\right) f_{j}\left(x_{j}(\tau)\right) d \tau .
\end{aligned}
$$

Thus, according to (13), one has

$$
\begin{aligned}
& \left|x_{i}(t)\right| \\
& \quad \leq\left|x_{i}(0)\right| E_{q, 1}\left(c_{i} t^{q}\right) \\
& \quad+\sum_{j=1}^{n}\left|a_{i j}\right| m_{j} \int_{0}^{t}(t-\tau)^{q-1} E_{q, q}\left(c_{i}(t-\tau)^{q}\right) d \tau .
\end{aligned}
$$

Noting that the Laplace transform of a Mittag-Leffler function is

$$
\int_{0}^{t} \tau^{\beta-1} E_{\beta_{1}, \beta_{2}}\left(-k \tau^{\beta_{1}}\right) d \tau=t^{\beta_{2}} E_{\beta_{1}, \beta_{2}+1}\left(-k t^{\beta_{1}}\right),
$$


then one has

$$
\left|x_{i}(t)\right| \leq\left|x_{i}(0)\right| E_{q, 1}\left(c_{i} t^{q}\right)+A_{i} t^{q} E_{q, q+1}\left(c_{i} t^{q}\right),
$$

where $A_{i}=\sum_{j=1}^{n}\left|a_{i j}\right| m_{j}$ is a positive constant.

It follows from Lemma 3 that

$$
A_{i} t^{q} E_{q, q+1}\left(-c_{i} t^{q}\right) \leq \frac{A_{i}}{-c_{i}} .
$$

Consequently, for large enough time $t$, one has

$$
\lim _{t \rightarrow \infty}\left|x_{i}(t)\right| \leq M
$$

where $M=\max _{1 \leq i \leq n}\left\{A_{i} / c_{i}\right\}$. This ends the proof of Theorem 4.

It should be pointed out that Theorem 4 can only drive $x_{i}(t)$ to a small region of zero. To discuss the asymptotic stability, one needs the following assumptions.

Assumption 5. The equilibrium point of system (11) is the origin.

Assumption 6. $f(x(t))$ is a Lipshitz continuous function; that is, the following inequality holds:

$$
\|f(x(t))-f(y(t))\| \leq l\|x(t)-y(t)\|,
$$

where $l>0$ is a Lipshitz constant.

Remark 7. It should be mentioned that Assumptions 5 and 6 are reasonable. In fact, every equilibrium point of system (11) can be moved to the origin by some linear transformations. In many FOCSs, the nonlinear functions are smooth and Lipshitz continuous, for example, fractional-order Lorenz system, fractional-order Chen system, fractional-order Lü system, fractional-order financial system, and fractional Volta system [45].
Theorem 8. Consider system (12). Under Assumption 6, if $(l b / c)\|A\|<q$, where $c=\max _{1 \leq i \leq n}-c_{i}$, then the asymptotical stability of system (12) can be guaranteed.

Proof. Suppose that $x(t), y(t) \in R^{n}$ are two arbitrary solutions of (12). Denote $e(t)=x(t)-y(t)$; then, one has

$$
\mathbb{D}^{q} e(t)=C e(t)+A(f(x(t))-f(y(t))) .
$$

It follows from (23) that

$$
\begin{aligned}
s^{q} E(s)= & s^{q-1} e(0)+C E(s) \\
& +A \mathscr{L}\{f(x(t))-f(y(t))\},
\end{aligned}
$$

where $E(s)=\mathscr{L}\{e(t)\}$.

After some straightforward manipulators, one has

$$
\begin{aligned}
E(s)= & \left(I s^{q}-C\right)^{-1} \\
& \cdot\left(s^{q-1} e(0)+A \mathscr{L}\{f(x(t))-f(y(t))\}\right) .
\end{aligned}
$$

Solving (25) yields

$$
\begin{aligned}
& e(t)=E_{q, 1}\left(C t^{q}\right) e(0)+A \int_{0}^{t}(t-\tau)^{q-1} \\
& \cdot E_{q, q}\left(C(t-\tau)^{q}\right) \mathscr{L}\{f(x(t))-f(y(t))\} d \tau .
\end{aligned}
$$

According to Assumption 6 and Lemma 1, one can find a constant $b>0$ such that

$$
\begin{aligned}
\|e(t)\| \leq & \frac{b\|e(0)\|}{1+\|C\| t^{q}} \\
& +l b\|A\| \int_{0}^{t} \frac{(t-\tau)^{q-1}}{1+\|C\|(t-\tau)^{q}}\|e(\tau)\| d \tau .
\end{aligned}
$$

Using Lemma 2, one has

$$
\begin{aligned}
\|x(t)\| & \leq \frac{b\|e(0)\|}{1+\|C\| t^{q}}+\int_{0}^{t} \frac{l b\|A\|(t-\tau)^{\alpha-1}\|e(0)\|}{\left(1+\|C\|(t-\tau)^{q}\right)\left(1+\|C\| \tau^{q}\right)} \exp \left(\int_{\tau}^{t} \frac{l b\|A\|(t-u)^{q-1}}{1+\|C\|(t-u)^{q}} d u\right) d \tau \\
& =\frac{b\|e(0)\|}{1+\|C\| t^{q}}+\int_{0}^{t} \frac{b l\|A\|(t-\tau)^{q-1}\|e(0)\|}{\left(1+\|C\| \tau^{q}\right)\left(1+\|C\|(t-\tau)^{q}\right)^{1-b / q\|C\|}} d \tau \\
& \leq \frac{b\|e(0)\|}{1+\|C\| t^{q}}+l b\|A\|\|e(0)\|\|C\|^{l b\|A\| / q\|C\|-2} \int_{0}^{t}(t-\tau)^{l b\|A\| /\|C\|-1} \tau^{-q} d \tau \\
& =\frac{b\|e(0)\|}{1+\|C\| t^{q}}+l b\|A\|\|e(0)\|\|C\|^{l b\|A\| / q\|C\|-2} \frac{\Gamma(l b\|A\| /\|C\|) \Gamma(1-q)}{\Gamma(1+l b\|A\| /\|C\|-q)} t^{l b\|A\| /\|C\|-q} .
\end{aligned}
$$

Noting that $(l b / c)\|A\|<q$, where $c=\|C\|=\max _{1 \leq i \leq n}-c_{i}$, then according to (28) one has

$$
\lim _{t \rightarrow \infty}\|e(t)\|=0,
$$

which completes the proof.
3.2. Synchronization Controller Design. The master and slave FOCSs are defined, respectively, as

$$
\begin{aligned}
& \mathbb{D}^{q} \zeta(t)=P \zeta(t)+Q \hbar(\zeta(t)), \\
& \mathbb{D}^{q \widehat{\zeta}(t)}=P \widehat{\zeta}(t)+Q \hbar(\widehat{\zeta}(t))+G u(t),
\end{aligned}
$$


where $\zeta(t), \widehat{\zeta}(t) \in \mathbb{R}^{n}$ are the state vectors of the master FOCS and slave FOCS, respectively, $P, Q, G \in \mathbb{R}^{n \times n}$ are three constant matrices, $G$ is a positive definite control gain matrix, and $u(t) \in \mathbb{R}^{n}$ represents the control input.

Define the synchronization error $\varepsilon(t)=\zeta(t)-\widehat{\zeta}(t)$. The objective of this section is to design a proper control input $u(t)$ such that $\varepsilon(t)$ converges to zero eventually. To proceed, let us give the following assumption first.

Assumption 9. $\hbar$ is a Lipshitz continuous function; that is, the following inequality holds:

$$
\|\hbar(\zeta(t))-\hbar(\widehat{\zeta}(t))\| \leq a_{0}\|\zeta(t)-\widehat{\zeta}(t)\|,
$$

where $a_{0}>0$ is a constant.

To meet the synchronization object, let us construct the following fractional-order sliding mode surface:

$$
\begin{aligned}
s(t)= & \Lambda \varepsilon(t) \\
& -\frac{1}{\Gamma(q)} \Lambda(P+K) \int_{0}^{t}(t-\tau)^{(q-1)} \varepsilon(\tau) d \tau,
\end{aligned}
$$

where $\Lambda, K \in \mathbb{R}^{n \times n}$ are two design matrices. Then, it follows from (30), (31), and (33) that

$$
\begin{aligned}
\mathbb{D}^{q} \mathcal{S}(t)= & \Lambda P \varepsilon(t)+\Lambda Q(\hbar(\zeta(t))-\hbar(\widehat{\zeta}(t)))-G u(t) \\
& -\Lambda(P+K) \varepsilon(t) \\
= & \Lambda Q(\hbar(\zeta(t))-\hbar(\widehat{\zeta}(t)))-G u(t) \\
& -\Lambda K \varepsilon(t) .
\end{aligned}
$$

Consequently, let $\mathbb{D}^{q} s(t)=0$; the control input can be given as

$$
u(t)=G^{-1} \Lambda Q(\hbar(\zeta(t))-\hbar(\widehat{\zeta}(t)))-G^{-1} \Lambda K \varepsilon(t) .
$$

Now, we can give the following results.

Theorem 10. Consider the master FOCS (30) and the slave FOCS (31) under Assumption 9. Suppose that the sliding surface is given by (33) and the control input is designed as (35). If the design matrices satisfy $P-\Lambda K<0$ and $a_{0}\|Q-\Lambda Q\| \leq \underline{\lambda} q$, where $\underline{\lambda}$ is the smallest eigenvalue of $\Lambda K-P$, then one can conclude that the synchronization error converges to the origin asymptotically.

Proof. It follows from (30) and (31) that

$$
\mathbb{D}^{q} \mathcal{\varepsilon}(t)=P \varepsilon(t)+Q \hbar(\hbar(\zeta(t))-\hbar(\widehat{\zeta}(t)))-G u(t) .
$$

Substituting (35) into (36) yields

$$
\begin{aligned}
\mathbb{D}^{q} \varepsilon(t)= & (P-\Lambda K) \varepsilon(t) \\
& +(Q-\Lambda Q) \hbar(\hbar(\zeta(t))-\hbar(\widehat{\zeta}(t))) .
\end{aligned}
$$

Noting that $P-\Lambda K<0$ and $a_{0}\|Q-\Lambda Q\| \leq \underline{\lambda} q$, it follows from (37), Assumption 9, and Theorem 8 that $\lim _{t \rightarrow \infty} \varepsilon(t)=0$. This completes the proof of Theorem 10.

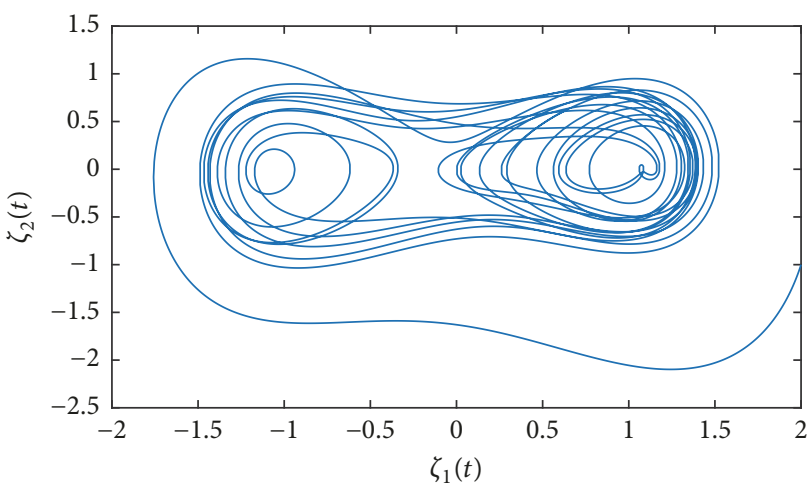

FIGURE 1: Phase attractor of FOCS (38).

\section{Simulation Results}

In this section, two examples will be given to show the effectiveness of the proposed method.

4.1. Synchronizing Two 2D Fractional-Order Duffing Systems. The fractional-order Duffing system is described by [46]

$$
\begin{aligned}
& \mathbb{D}^{q} \zeta_{1}(t)=\zeta_{2}(t), \\
& \mathbb{D}^{q} \zeta_{2}(t)=\zeta_{1}(t)-\zeta_{1}^{3}(t)-0.15 \zeta_{2}(t)+0.3 \cos (t) .
\end{aligned}
$$

The Jacobian matrix of system (38) for the equilibrium point $E_{1}^{*}=\left(\zeta_{1}^{*}, \zeta_{2}^{*}\right)$ is

$$
J_{E^{*}}=\left[\begin{array}{cc}
0 & 1 \\
1-3 \zeta_{1}^{*} & -0.15
\end{array}\right] .
$$

It is easy to know that system (38) has three equilibria: $E_{1}=(1.0729,0), E_{2}=(-0.9062,0)$, and $E_{3}=(-0.1667,0)$. For equilibrium $E_{1}$, we get the eigenvalues $\lambda_{1} \approx-0.0750+$ $1.4876 i$ and $\lambda_{2} \approx-0.0750-1.4876 i$. For equilibrium $E_{2}$, the eigenvalues are $\lambda_{1} \approx 1.8548$ and $\lambda_{2} \approx-2.0048$. For equilibrium $E_{3}$, we obtain the eigenvalues $\lambda_{1} \approx 1.1521$ and $\lambda_{2} \approx-1.3021$. According to these eigenvalues, we can conclude that a minimal commensurate order to obtain the chaotic behavior of system (38) is [45]

$$
q>\frac{2}{\pi} \arctan \left(\frac{1.4876}{0.0750}\right)=0.9679 .
$$

Under the initial conditions $\zeta_{1}(0)=2$ and $\zeta_{2}(0)=-1$ and the fractional order $q=0.98$, FOCS (38) shows a chaotic behavior, which is depicted in Figure 1.

According to (30) and (38), it is easy to know that

$$
\begin{aligned}
P & =\left[\begin{array}{ll}
0 & 1 \\
1 & 0
\end{array}\right], \\
Q & =\left[\begin{array}{ll}
0 & 0 \\
0 & 1
\end{array}\right], \\
\hbar(t) & =\left[\begin{array}{c}
0 \\
-\zeta_{1}^{3}(t)-0.15 \zeta_{2}(t)+0.3 \cos (t)
\end{array}\right] .
\end{aligned}
$$




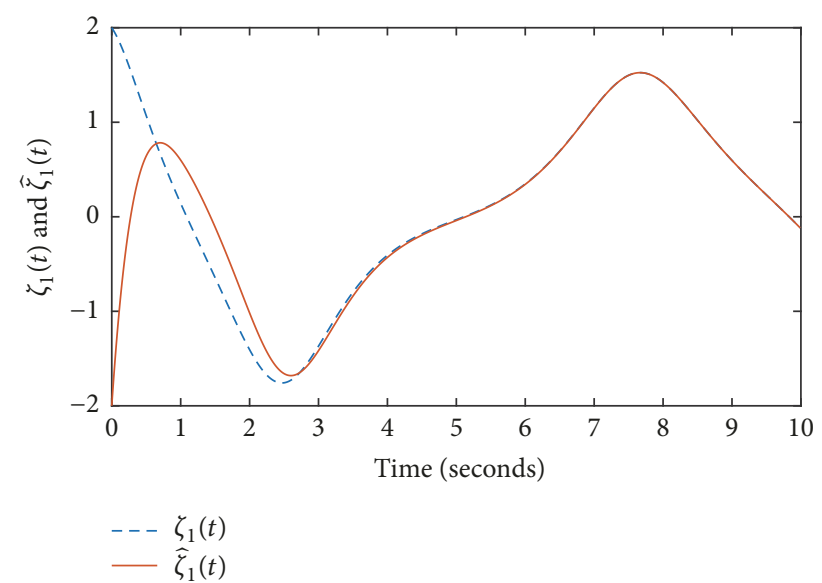

Figure 2: Synchronization between $\zeta_{1}(t)$ and $\widehat{\zeta}_{1}(t)$.

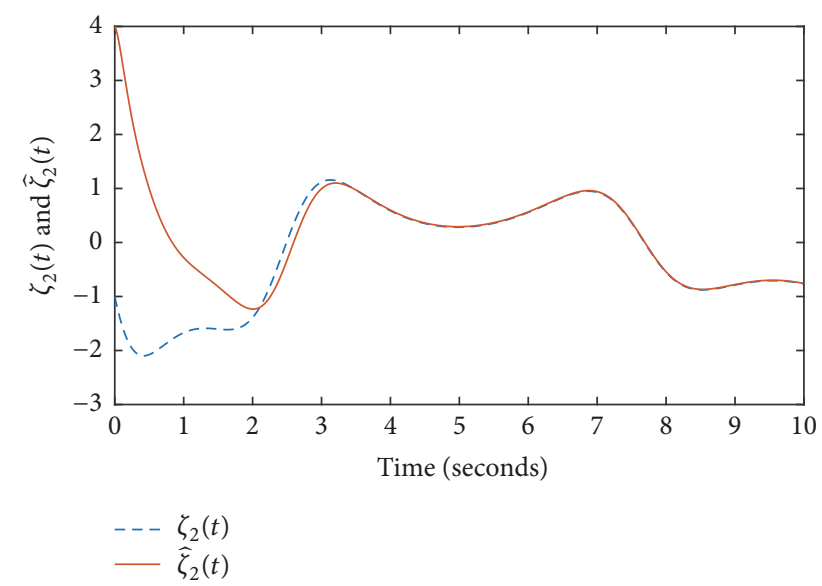

FIGURE 3: Synchronization between $\zeta_{2}(t)$ and $\widehat{\zeta}_{2}(t)$.

Since system (38) is a chaotic system, then we know that both signals $\zeta_{1}(t)$ and $\zeta_{2}(t)$ are bounded (from Figure 1, one knows that $\left|\zeta_{1}(t)\right|<2$ and $\left.\left|\zeta_{2}(t)\right|<2\right)$. Thus, Assumption 9 is satisfied with $a_{0}=3$.

In the simulation, the initial condition for the slave FOCS is $\widehat{\zeta}_{1}(0)=-2$ and $\widehat{\zeta}_{2}(0)=4$. Suppose that $G=\operatorname{diag}(1,1)$. The design matrices are chosen as

$$
\begin{aligned}
& K=\left[\begin{array}{cc}
-73 & 34 \\
52 & -12
\end{array}\right], \\
& \Lambda=\left[\begin{array}{ll}
0.1 & 0.2 \\
0.2 & 0.3
\end{array}\right] .
\end{aligned}
$$

Thus, we have that $\|Q-\Lambda Q\|=0.728, \underline{\lambda}=3.1$, and the two conditions $P-\Lambda K<0$ and $a_{0}\|Q-\Lambda Q\| \leq \underline{\lambda} q$ in Theorem 10 are satisfied.

The simulation results are presented in Figures 2-5. The results where the state variables of the slave FOCS track the master system's states are presented in Figures 2 and 3. The time response of the synchronization errors is depicted in Figure 4. From these pictures, we can see that the synchronization controller works well, and the synchronization

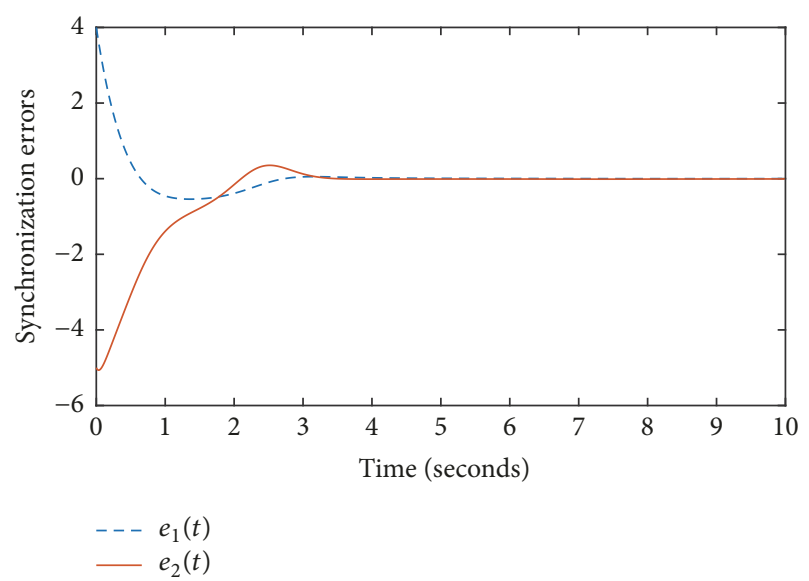

FIgURE 4: Time response of synchronization errors $e_{1}(t)$ and $e_{2}(t)$.

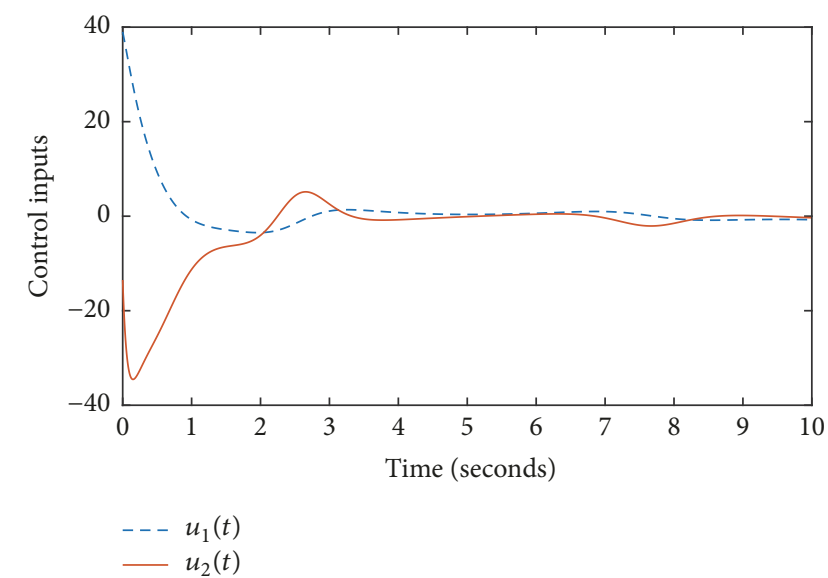

FIGURE 5: Time response of control inputs $u_{1}(t)$ and $u_{2}(t)$.

errors converge to the origin fast. From (35), we know that the synchronization control input is a continuous function. The smoothness of the control input is given in Figure 5, from which we can see that the proposed controller has small fluctuation.

4.2. Synchronizing Two 3D Fractional-Order Chaotic Neural Networks. Let us consider the following fractional-order chaotic neural networks expressed by [15]

$$
\begin{aligned}
\mathbb{D}^{q} \zeta_{1}(t)= & -\zeta_{1}(t)+2 \tanh \left(\zeta_{1}(t)\right)-1.2 \tanh \left(\zeta_{2}(t)\right) \\
\mathbb{D}^{9} \zeta_{2}(t)= & -\zeta_{2}(t)+2 \tanh \left(\zeta_{1}(t)\right)+1.71 \tanh \left(\zeta_{2}(t)\right) \\
& +1.15 \tanh \left(\zeta_{3}(t)\right) \\
\mathbb{D}^{9} \zeta_{3}(t)= & -\zeta_{3}(t)-4.75 \tanh \left(\zeta_{1}(t)\right) \\
& +1.10 \tanh \left(\zeta_{3}(t)\right) .
\end{aligned}
$$

Suppose that $q=0.95$ and the initial condition is $\zeta_{1}(0)=$ $-0.3, \zeta_{2}(0)=0.4$, and $\zeta_{3}(0)=0.3$. The dynamical behavior of FOCS (43) is given in Figure 6. 


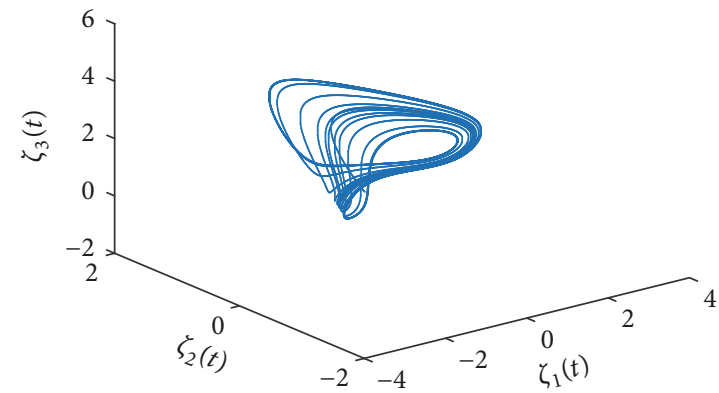

(a)

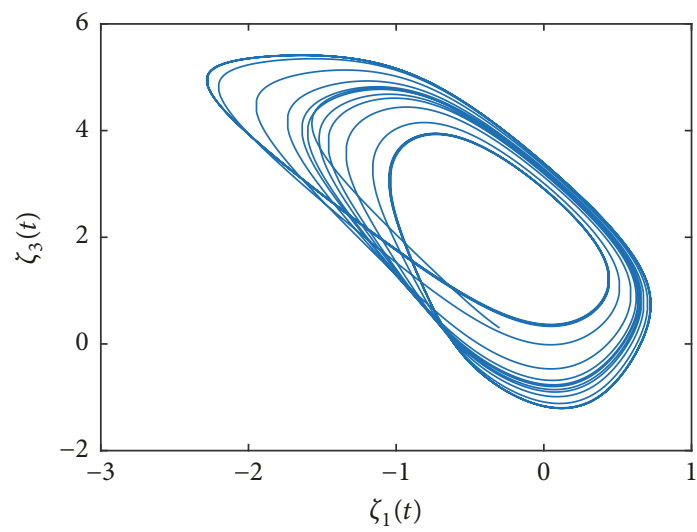

(c)

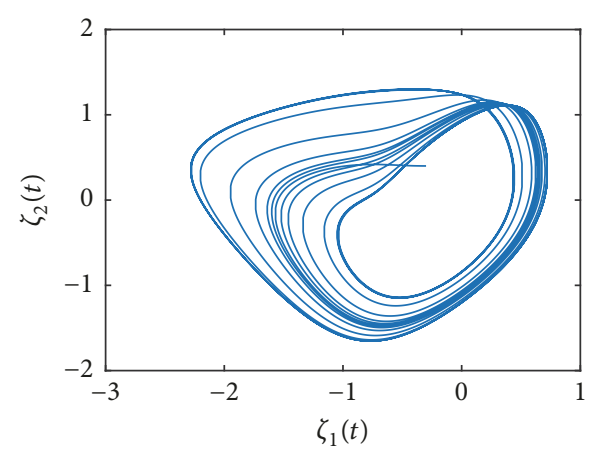

(b)

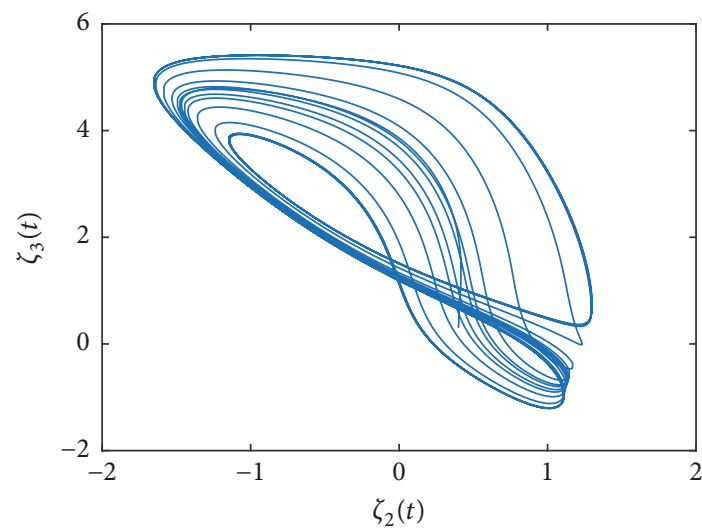

(d)

Figure 6: Dynamical behavior of system (43) in (a) 3D space, (b) $\zeta_{1}(t)-\zeta_{2}(t)$ plane, (c) $\zeta_{1}(t)-\zeta_{3}(t)$ plane, and (d) $\zeta_{2}(t)-\zeta_{3}(t)$ plane.

It is easy to know in the master chaotic system (43) that $P=\operatorname{diag}(-1,-1,-1), \hbar(t)=$ $\left[\tanh \left(\zeta_{1}(t)\right), \tanh \left(\zeta_{2}(t)\right), \tanh \left(\zeta_{3}(t)\right)\right]^{T}$,

$$
Q=\left[\begin{array}{ccc}
2.00 & -1.20 & 0.00 \\
2.00 & 1.71 & 1.15 \\
-4.75 & 0.00 & 1.10
\end{array}\right]
$$

Thus, we have $\|Q\|=1.235$ and $\hbar(t)$ satisfy the Lipshitz condition. The Lipshitz constant $a_{0}$ can be chosen as 1 .

The initial condition of the slave FOCS is $\widehat{\zeta}_{1}(0)=$ $3.2, \widehat{\zeta}_{2}(0)=-4$, and $\widehat{\zeta}_{3}(0)=-3.5$. Let $G=\operatorname{diag}(1,1,1)$. The design matrices are chosen as

$$
\begin{aligned}
K & =\left[\begin{array}{ccc}
1.2484 & -0.1401 & 0.0127 \\
0.0127 & 1.1210 & -1.1019 \\
-0.1146 & -0.0892 & 0.9172
\end{array}\right], \\
\Lambda & =\left[\begin{array}{ccc}
0.8 & 0.1 & 0 \\
0 & 0.9 & 0.1 \\
0.1 & 0.1 & 1.1
\end{array}\right] .
\end{aligned}
$$

Thus, we know that $\|Q-\Lambda Q\|=0.7115, \underline{\lambda}=2$, and the two conditions $P-\Lambda K<0$ and $a_{0}\|Q-\Lambda Q\| \leq \underline{\lambda} q$ in Theorem 10 are satisfied.

The simulation results are given in Figures 7 and 8 . Just like the results in Figures 2-5, we know that good synchronization performance has been obtained.

\section{Conclusion}

In this paper, two stability criteria for fractional-order nonlinear systems are given. Based on these theorems, the synchronization of two identical FOCSs is addressed. A fractional-order sliding surface, which contains a fractionalorder integral of the synchronization errors, is given. The proposed controller can guarantee the asymptotical stability of the closed-loop systems. However, in the controller design, we need to know the exact value of the Lipchitz constant. How to reduce this condition is one of our future research directions.

\section{Conflicts of Interest}

The author does not have a direct financial relation with any commercial identity mentioned in this paper that might lead to conflicts of interest. 


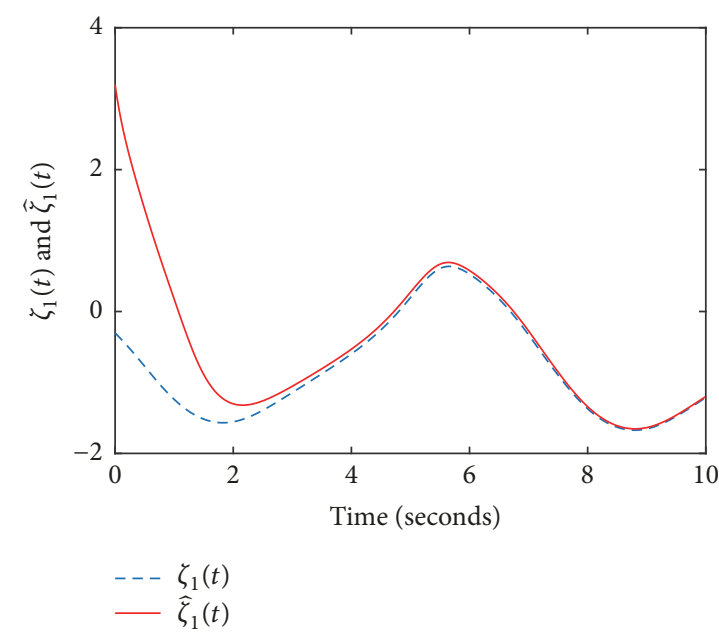

(a)

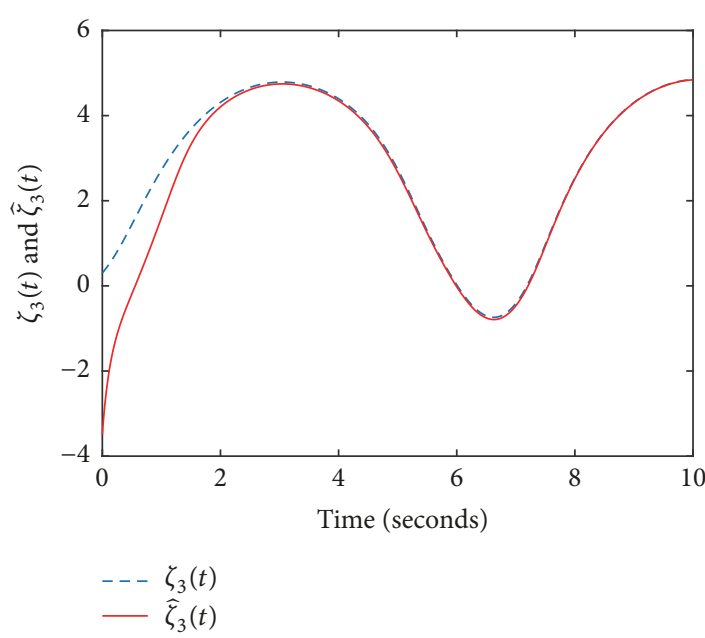

(c)

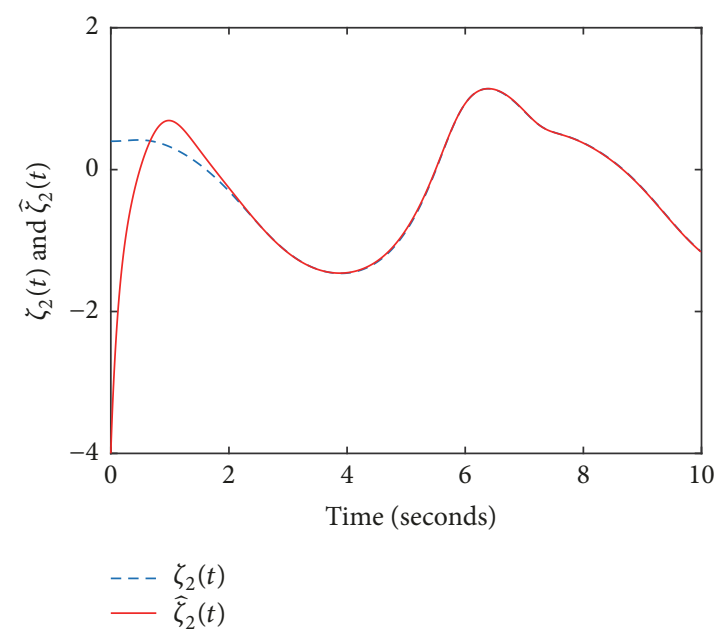

(b)

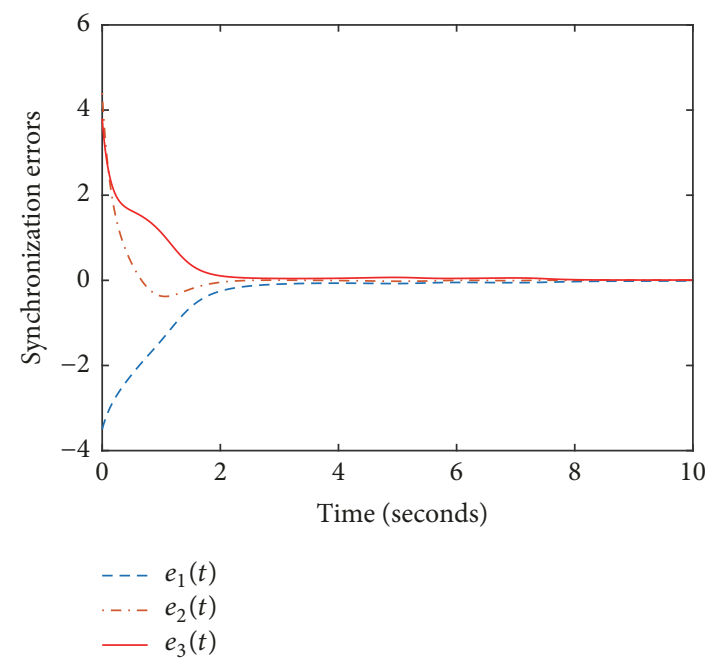

(d)

FIGURE 7: Simulation results in (a) synchronization between $\zeta_{1}(t)$ and $\widehat{\zeta}_{1}(t)$, (b) synchronization between $\zeta_{2}(t)$ and $\widehat{\zeta}_{2}(t)$, (c) synchronization between $\zeta_{3}(t)$ and $\widehat{\zeta}_{3}(t)$, and (d) synchronization errors $e_{1}(t), e_{2}(t)$, and $e_{3}(t)$.

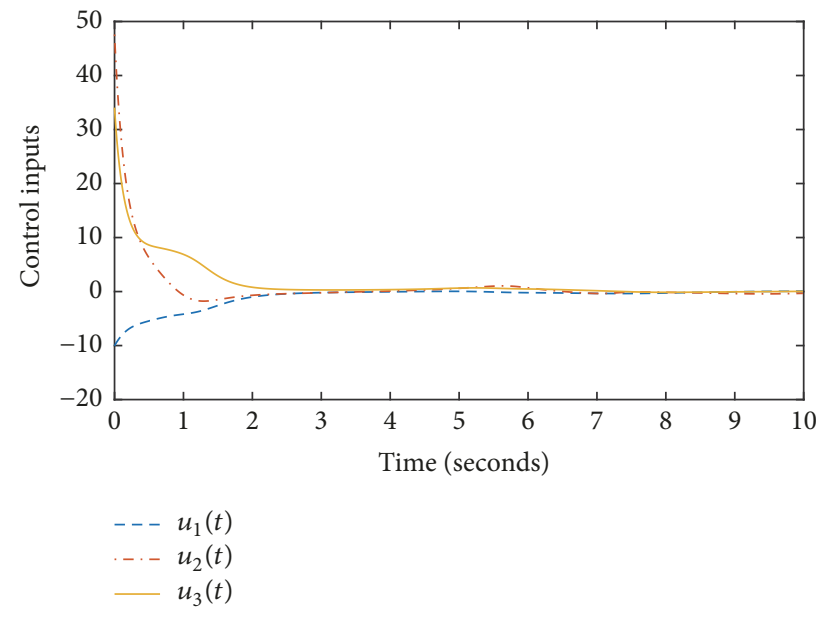

FIGURE 8: Time response of control inputs $u_{1}(t), u_{2}(t)$, and $u_{3}(t)$.

\section{Acknowledgments}

This work is supported by the National Natural Science Foundation of China (Grant no. 11302184) and the Young and Middle-Aged Teacher Education and Science Research Foundation of Fujian Province of China (Grant no. JAT170423).

\section{References}

[1] H. Liu, S.-G. Li, Y.-G. Sun, and H.-X. Wang, "Adaptive fuzzy synchronization for uncertain fractional-order chaotic systems with unknown non-symmetrical control gain," Wuli Xuebao/Acta Physica Sinica, vol. 64, no. 7, Article ID 070503, 2015.

[2] H. Liu, S. Li, Y. Sun, and H. Wang, "Prescribed performance synchronization for fractional-order chaotic systems," Chinese Physics B, vol. 24, no. 9, Article ID 090505, 2015.

[3] S. Vaidyanathan and S. Pakiriswamy, "A five-term 3-D novel conservative chaotic system and its generalized projective 
synchronization via adaptive control method," Control Theory and Technology, vol. 9, no. 1, pp. 61-78, 2016.

[4] C. Volos, V.-T. Pham, E. Zambrano-Serrano, J. M. MunozPacheco, S. Vaidyanathan, and E. Tlelo-Cuautle, "Analysis of a 4-D hyperchaotic fractional-order memristive system with hidden attractors," in Advances in Memristors, Memristive Devices and Systems, vol. 701, pp. 207-235, Springer, 2017.

[5] C. Volos, S. Vaidyanathan, V.-T. Pham et al., "Adaptive control and synchronization of a memristor-based Shinriki's system," in Advances in Memristors, Memristive Devices and Systems, vol. 701, pp. 237-261, Springer, 2017.

[6] A. Ouannas, Z. Odibat, N. Shawagfeh, A. Alsaedi, and B. Ahmad, "Universal chaos synchronization control laws for general quadratic discrete systems," Applied Mathematical Modelling: Simulation and Computation for Engineering and Environmental Systems, vol. 45, pp. 636-641, 2017.

[7] A. Ouannas and Z. Odibat, "On inverse generalized synchronization of continuous chaotic dynamical systems," International Journal of Applied and Computational Mathematics, vol. 2, no. 1, pp. 1-11, 2016.

[8] A. Boulkroune, A. Bouzeriba, T. Bouden, and A. T. Azar, "Fuzzy adaptive synchronization of uncertain fractional-order chaotic systems," in Advances in chaos theory and intelligent control, vol. 337, pp. 681-697, Springer, 2016.

[9] A. Boulkroune, A. Bouzeriba, and T. Bouden, "Fuzzy generalized projective synchronization of incommensurate fractionalorder chaotic systems," Neurocomputing, vol. 173, pp. 606-614, 2016.

[10] S. Çiçek, A. Ferikoglu, and I. Pehlivan, "A new 3D chaotic system: dynamical analysis, electronic circuit design, active control synchronization and chaotic masking communication application," Optik - International Journal for Light and Electron Optics, vol. 127, no. 8, pp. 4024-4030, 2016.

[11] S. Vaidyanathan and A. T. Azar, "Dynamic analysis, adaptive feedback control and synchronization of an eight-term 3-D novel chaotic system with three quadratic nonlinearities," in Advances in Chaos Theory and Intelligent Control, vol. 337, pp. 155-178, Springer, 2016.

[12] S. Sampath and S. Vaidyanathan, "Hybrid synchronization of identical chaotic systems via novel sliding control method with application to Sampath four-scroll chaotic system," Control Theory and Technology, vol. 9, no. 1, pp. 221-235, 2016.

[13] P. Muthukumar, P. Balasubramaniam, and K. Ratnavelu, "Sliding mode control design for synchronization of fractional order chaotic systems and its application to a new cryptosystem," International Journal of Dynamics and Control, vol. 5, no. 1, pp. 115-123, 2017.

[14] H. Liu, S. Li, H. Wang, Y. Huo, and J. Luo, "Adaptive synchronization for a class of uncertain fractional-order neural networks," Entropy, vol. 17, no. 10, pp. 7185-7200, 2015.

[15] H. Liu, Y. Pan, S. Li, and Y. Chen, "Synchronization for fractional-order neural networks with full/under-actuation using fractional-order sliding mode control," International Journal of Machine Learning and Cybernetics, pp. 1-14, 2017.

[16] H. Liu, S.-G. Li, H.-X. Wang, and G.-J. Li, "Adaptive fuzzy synchronization for a class of fractional-order neural networks," Chinese Physics B, vol. 26, no. 3, Article ID 030504, 2017.

[17] H. Li, J. Yu, C. Hilton, and H. Liu, "Adaptive sliding-mode control for nonlinear active suspension vehicle systems using TS fuzzy approach," IEEE Transactions on Industrial Electronics, vol. 60, no. 8, pp. 3328-3338, 2013.
[18] Y. Pan and H. Yu, "Composite learning from adaptive dynamic surface control," Institute of Electrical and Electronics Engineers Transactions on Automatic Control, vol. 61, no. 9, pp. 2603-2609, 2016.

[19] H. Li, P. Shi, and D. Yao, "Adaptive sliding mode control of markov jump nonlinear systems with actuator faults," IEEE Transactions on Automatic Control, vol. 62, no. 4, pp. 1933-1939, 2016.

[20] H. Wang, "Core-EP decomposition and its applications," Linear Algebra and Its Applications, vol. 508, pp. 289-300, 2016.

[21] H. Wang and X. Liu, "A partial order on the set of complex matrices with index one," Linear and Multilinear Algebra, pp. 1-11, 2017.

[22] J. Liu, S. Vazquez, L. Wu, A. Marquez, H. Gao, and L. G. Franquelo, "Extended State Observer-Based Sliding-Mode Control for Three-Phase Power Converters," IEEE Transactions on Industrial Electronics, vol. 64, no. 1, pp. 22-31, 2017.

[23] Y. Pan, C. Yang, L. Pan, and H. Yu, "Integral Sliding Mode Control: Performance, Modification and Improvement," IEEE Transactions on Industrial Informatics, 2017.

[24] H. Wang and W. Guo, "The minimal rank of matrix expressions with respect to Hermitian matrix-revised," Journal of The Franklin Institute, vol. 353, no. 5, pp. 1206-1219, 2016.

[25] H. Komurcugil and S. Biricik, "Time-Varying and Constant Switching Frequency-Based Sliding-Mode Control Methods for Transformerless DVR Employing Half-Bridge VSI," IEEE Transactions on Industrial Electronics, vol. 64, no. 4, pp. 25702579, 2017.

[26] Y. Pan, H. Wang, X. Li, and H. Yu, "Adaptive CommandFiltered Backstepping Control of Robot Arms With Compliant Actuators," IEEE Transactions on Control Systems Technology, pp. 1-8, 2017.

[27] Y. Pan, Z. Guo, X. Li, and H. Yu, "Output-feedback adaptive neural control of a compliant differential SMA actuator," IEEE Transactions on Control Systems Technology, vol. 25, no. 6, pp. 2202-2210, 2017.

[28] C. Yin, S. Dadras, S.-M. Zhong, and Y. Chen, "Control of a novel class of fractional-order chaotic systems via adaptive sliding mode control approach," Applied Mathematical Modelling, vol. 37, no. 4, pp. 2469-2483, 2013.

[29] C. Yin, Y. Cheng, S.-M. Zhong, and Z. Bai, "Fractionalorder switching type control law design for adaptive sliding mode technique of 3D fractional-order nonlinear systems," Complexity, vol. 21, no. 6, pp. 363-373, 2016.

[30] A. Mohammadzadeh and S. Ghaemi, "A modified sliding mode approach for synchronization of fractional-order chaotic/hyperchaotic systems by using new self-structuring hierarchical type-2 fuzzy neural network," Neurocomputing, vol. 191, pp. 200-213, 2016.

[31] J. Shen and J. Lam, "Non-existence of finite-time stable equilibria in fractional-order nonlinear systems," Automatica, vol. 50, no. 2, pp. 547-551, 2014.

[32] J. C. Trigeassou, N. Maamri, J. Sabatier, and A. Oustaloup, "A Lyapunov approach to the stability of fractional differential equations," Signal Processing, vol. 91, no. 3, pp. 437-445, 2011.

[33] D. Matignon, "Stability result for fractional differential equations with applications to control processing," in Computational Engineering in Systems Applications, pp. 963-968, IMACS, IEEE-SMC, Lille, France, 1997.

[34] J.-G. Lu and G. Chen, "Robust stability and stabilization of fractional-order interval systems: an LMI approach," IEEE 
Transactions on Automatic Control, vol. 54, no. 6, pp. 1294-1299, 2009.

[35] Y. Li, Y. Chen, and I. Podlubny, "Mittag-Leffler stability of fractional order nonlinear dynamic systems," Automatica, vol. 45, no. 8, pp. 1965-1969, 2009.

[36] H. Delavari, D. Baleanu, and J. Sadati, "Stability analysis of Caputo fractional-order nonlinear systems revisited," Nonlinear Dynamics, vol. 67, no. 4, pp. 2433-2439, 2012.

[37] X.-J. Wen, Z.-M. Wu, and J.-G. Lu, "Stability analysis of a class of nonlinear fractional-order systems," IEEE Transactions on Circuits and Systems II: Express Briefs, vol. 55, no. 11, pp. 11781182, 2008.

[38] S. Liu, W. Jiang, X. Li, and X.-F. Zhou, "Lyapunov stability analysis of fractional nonlinear systems," Applied Mathematics Letters, vol. 51, pp. 13-19, 2016.

[39] X. Yang, C. Li, T. Huang, and Q. Song, "Mittag-Leffler stability analysis of nonlinear fractional-order systems with impulses," Applied Mathematics and Computation, vol. 293, pp. 416-422, 2017.

[40] H. Liu, S. Li, J. D. Cao, A. G. Alsaedi, and F. E. Alsaadi, "Adaptive fuzzy prescribed performance controller design for a class of uncertain fractional-order nonlinear systems with external disturbances," Neurocomputing, vol. 219, pp. 422-430, 2017.

[41] H. Liu, Y. Pan, S. Li, and Y. Chen, "Adaptive Fuzzy Backstepping Control of Fractional-Order Nonlinear Systems," IEEE Transactions on Systems, Man, and Cybernetics: Systems, vol. 47, no. 8, pp. 2209-2217, 2017.

[42] I. Podlubny, Fractional Differential Equations, vol. 198 of Mathematics in Science and Engineering, Academic Press, San Diego, Calif, USA, 1999.

[43] L. Chen, Y. Chai, R. Wu, and J. Yang, "Stability and stabilization of a class of nonlinear fractional-order systems with caputo derivative," IEEE Transactions on Circuits and Systems II: Express Briefs, vol. 59, no. 9, pp. 602-606, 2012.

[44] H. Liu, S. Li, G. Li, and H. Wang, "Adaptive Controller Design for a Class of Uncertain Fractional-Order Nonlinear Systems: An Adaptive Fuzzy Approach," International Journal of Fuzzy Systems, pp. 1-14, 2017.

[45] I. Petráš, Fractional-Order Nonlinear Systems: Modeling, Analysis and Simulation, Springer Science \& Business Media, 2011.

[46] Z. M. Ge and C. Y. Ou, "Chaos in a fractional order modified Duffing system," Chaos, Solitons \& Fractals, vol. 34, no. 2, pp. 262-291, 2007. 


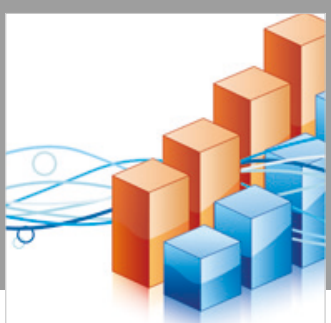

Advances in

Operations Research

\section{-n-m}
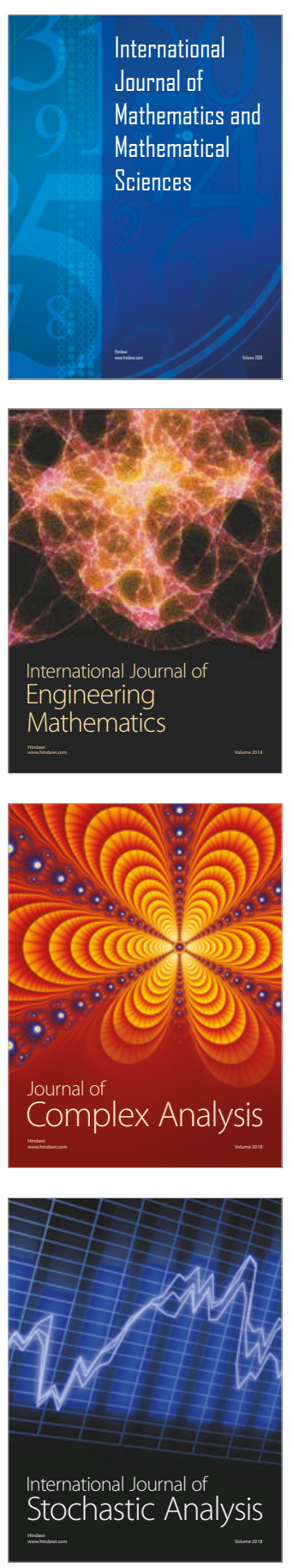
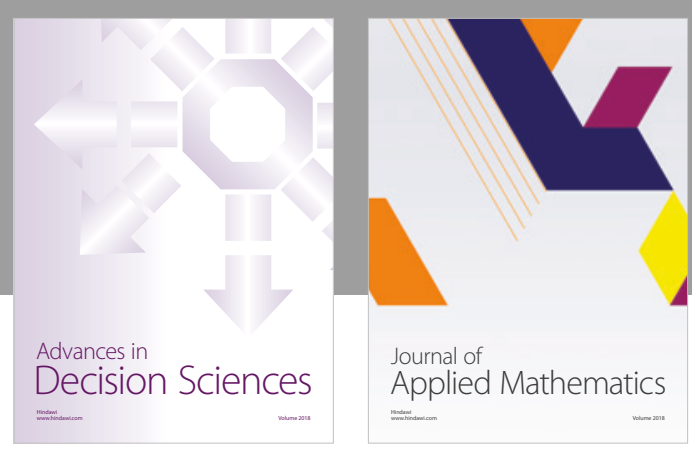

Journal of

Applied Mathematics
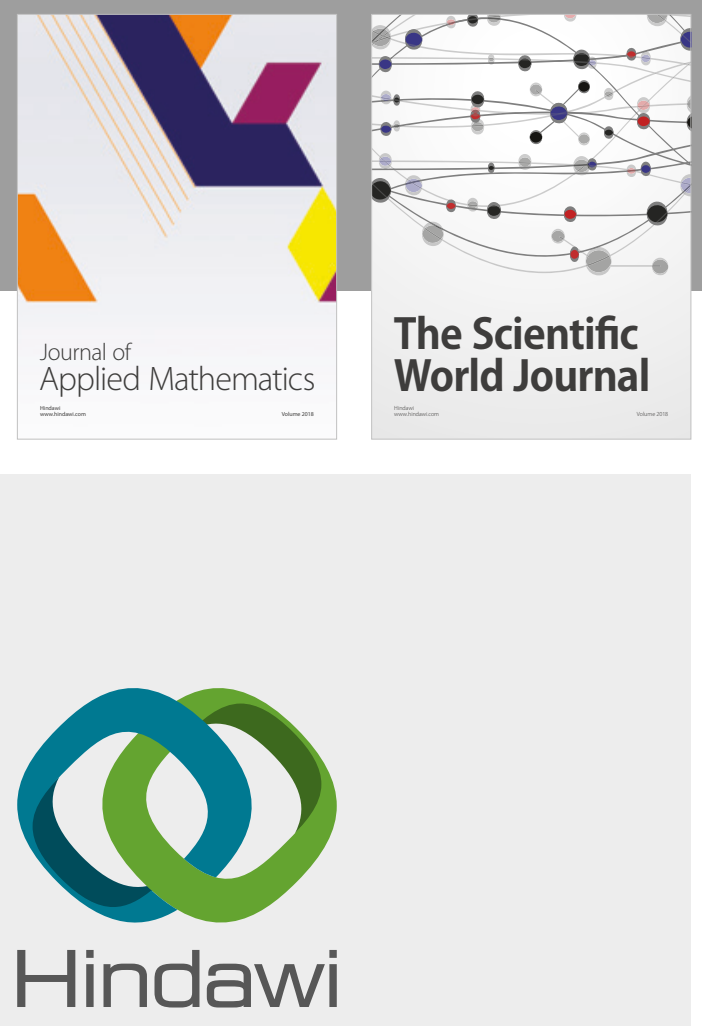

Submit your manuscripts at

www.hindawi.com

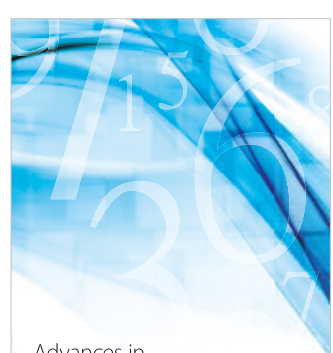

Advances in
Numerical Analysis
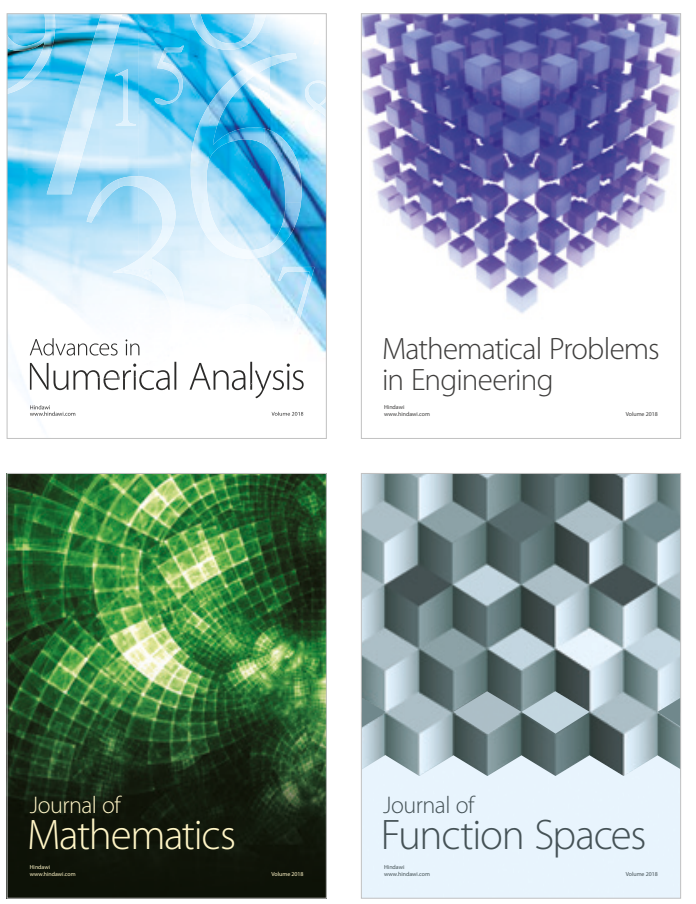

Mathematical Problems in Engineering

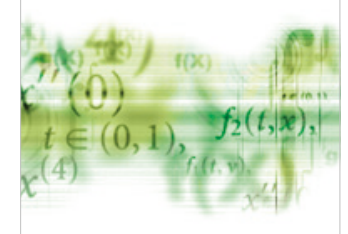

International Journal of

Differential Equations

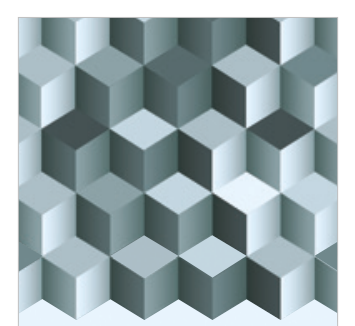

Journal of

Function Spaces

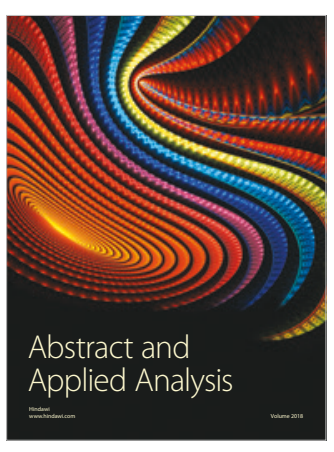

The Scientific

World Journal

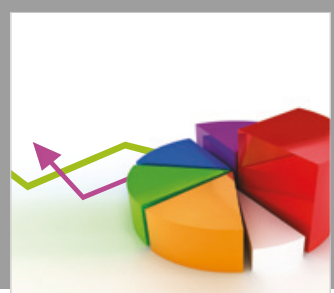

Journal of

Probability and Statistics
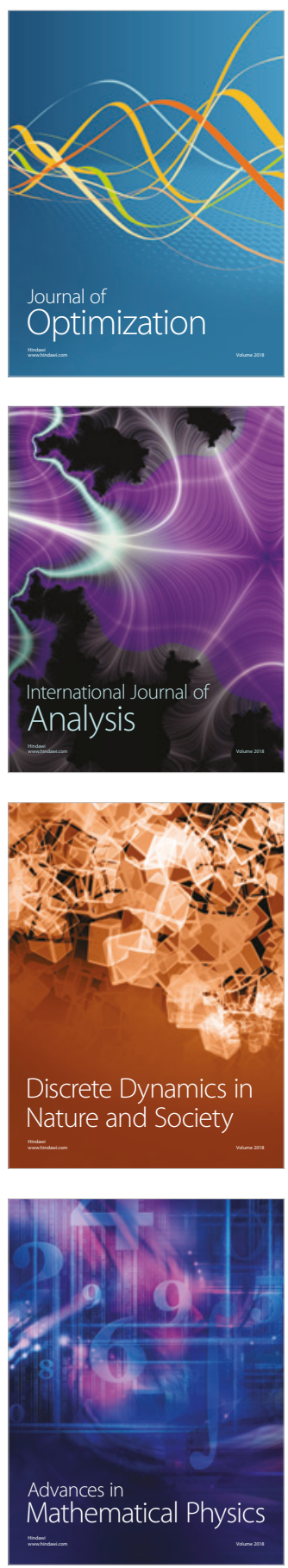